\title{
Towards a solution of the cosmological constant problem
}

\author{
F.R. Klinkhamer $\# 1)$ and G.E. Volovik ${ }^{*+1)}$ \\ \# Institute for Theoretical Physics, University of Karlsruhe, Karlsruhe Institute of Technology, 76128 Karlsruhe, Germany \\ * Low Temperature Laboratory, Aalto University, FI-00076 AALTO, Finland \\ + Landau Institute for Theoretical Physics RAS, Kosygina 2, 119334 Moscow, Russia
}

Submitted January 25, 2010

\begin{abstract}
The standard model of elementary particle physics and the theory of general relativity can be extended by the introduction of a vacuum variable which is responsible for the near vanishing of the present cosmological constant (vacuum energy density). The explicit realization of this vacuum variable can be via a three-form gauge field, an aether-type velocity field, or any other field appropriate for the description of the equilibrium state corresponding to the Lorentz-invariant quantum vacuum. The extended theory has, without fine-tuning, a Minkowski-type solution of the field equations with spacetime-independent fields and provides, therefore, a possible solution of the main cosmological constant problem.
\end{abstract}

PACS: 04.20.Cv, 98.80.Es, 95.36.+x

\section{INTRODUCTION}

The main cosmological constant problem is to understand why, naturally, the quantum-mechanical zeropoint energy of the vacuum does not produce a large cosmological constant or, in other words, to discover the way the zero-point energy is canceled without finetuning the theory. Restricting to established physics, this problem was formulated by Weinberg in the following pragmatic way [1, 2]: how to find an extension of the standard model of elementary particle physics and the theory of general relativity, for which there exists, without fine-tuning, a Minkowski-spacetime solution with spacetime-independent fields.

An adjustment-type solution of the cosmological constant problem appears, however, to be impossible with a fundamental scalar field and Weinberg writes in the last sentence of Sec. 2 in Ref. 2 that, to the best of his knowledge, "no one has found a way out of this impasse." In this Letter, we present a way around the impasse, which employs a quantity $q$ that acts as a selfadjusting scalar field but is non-fundamental [3, 4, 5].

The main goal of the present publication is to describe, in a more or less consistent way, a particular theoretical framework for addressing the cosmological constant problem. Obviously, this builds on previous work of the present authors and many others (see citations below). But there are also two important new results, which will be indicated explicitly.

\footnotetext{
1) e-mail: frans.klinkhamer@kit.edu, volovik@boojum.hut.fi
}

\section{MINKOWSKI EQUILIBRIUM VACUUM}

Our discussion starts from the theory outlined in Ref. 4. We introduce a special quantity, the vacuum "charge" $q$, to describe the statics of the quantum vacuum. A concrete example of this vacuum variable is given by the four-form field strength [6, 7, 8, 9, 10, 11, 12, 13, 14, expressed in terms of $q$ as $F_{\alpha \beta \gamma \delta}=$ $q \sqrt{-\operatorname{det} g} \epsilon_{\alpha \beta \gamma \delta}$ (see below for further details). This particular vacuum variable $q$ is associated with an energy scale $E_{\mathrm{UV}}$ that is assumed to be much larger than the electroweak energy scale $E_{\text {ew }} \sim 10^{3} \mathrm{GeV}$ and possibly to be of the order of the gravitational energy scale $E_{\text {Planck }} \equiv 1 / \sqrt{8 \pi G_{N}} \approx 2.44 \times 10^{18} \mathrm{GeV}$. Here, and in the following, natural units are used with $\hbar=c=1$.

Specifically, the effective action of our theory is given by

$$
\begin{aligned}
S^{\mathrm{eff}}[A, g, \psi]= & -\int_{\mathbb{R}^{4}} d^{4} x \sqrt{-\operatorname{det} g}(K(q) R[g] \\
& \left.+\epsilon(q)+\mathcal{L}_{\mathrm{SM}}^{\mathrm{eff}}[\psi, g]\right) \\
F_{\alpha \beta \gamma \delta} \equiv & q \epsilon_{\alpha \beta \gamma \delta} \sqrt{-\operatorname{det} g}=\nabla_{[\alpha} A_{\beta \gamma \delta]}, \\
q^{2}= & -\frac{1}{24} F_{\alpha \beta \gamma \delta} F^{\alpha \beta \gamma \delta}
\end{aligned}
$$

where $R$ denotes the Ricci curvature scalar, $\epsilon_{\alpha \beta \gamma \delta}$ the Levi-Civita tensor density, $\nabla_{\alpha}$ the covariant derivative, and the square bracket around spacetime indices complete anti-symmetrization. Throughout, we use the same conventions as in Ref. 1], in particular, those for the Riemann curvature tensor and the metric signature $(-+++)$.

The vacuum energy density $\epsilon$ in (1a) depends on the 
vacuum variable $q=q[A, g]$ and the same is assumed to hold for the gravitational coupling parameter $K$. The single field $\psi$ combines all the fields of the standard model (spinor, gauge, Higgs, and ghost fields [15]) and, for simplicity, the scalar Lagrange density $\mathcal{L}_{\mathrm{SM}}^{\mathrm{eff}}$ in (1a) is taken to be without direct $q$ dependence. The original standard model fields collected in $\psi(x)$ are quantum fields with vanishing vacuum expectation values in Minkowski spacetime (this holds, in particular, for the physical Higgs field $H(x)$ [15]). The effective action takes $\psi(x)$ to be a classical field, but has additional terms to reflect the quantum effects [16]. The metric field $g_{\alpha \beta}(x)$ and the three-form gauge field $A_{\beta \gamma \delta}(x)$ [or other $q$-related fields discussed later on] are, for the moment, considered to be genuine classical fields.

The setup, now, is such that a possible constant term $\Lambda_{\mathrm{SM}}$ in $\mathcal{L}_{\mathrm{SM}}^{\mathrm{eff}}$ (which includes the zero-point energies from the standard model fields) has been absorbed in $\epsilon(q)$, so that, in the end, $\mathcal{L}_{\mathrm{SM}}^{\mathrm{eff}}[\psi, g]$ contains only $\psi$-dependent terms, with the metric $g_{\alpha \beta}$ (or vierbein $e_{\alpha}^{a}$ ) entering through the usual covariant derivatives. In short, the following holds true:

$$
\mathcal{L}_{\mathrm{SM}}^{\mathrm{eff}}\left[\psi_{0}, \eta\right]=0
$$

where $\psi_{0}$ denotes the constant values for the standard model fields over Minkowski spacetime and $\eta$ stands for the Minkowski metric $\eta_{\alpha \beta}=\operatorname{diag}(-1,1,1,1)$ in standard coordinates.

The actual spectrum of the vacuum energy density (meaning the different contributions to $\epsilon$ from different energy scales) is not important for the cancellation mechanism to be discussed in this Letter. Still, we assume, for definiteness, that the vacuum energy density $\epsilon(q)$ splits into a constant part and a variable part:

$$
\epsilon(q)=\Lambda_{\text {bare }}+\epsilon_{\mathrm{var}}(q) \equiv \Lambda_{\mathrm{SM}}+\Lambda_{\mathrm{UV}}+\epsilon_{\mathrm{var}}(q),
$$

with $\partial \epsilon_{\mathrm{var}} / \partial q \neq 0$, a constant term $\Lambda_{\mathrm{SM}}$ of typical size $\left|\Lambda_{\mathrm{SM}}\right| \sim\left(E_{\text {ew }}\right)^{4}$ removed from $\mathcal{L}_{\mathrm{SM}}^{\mathrm{eff}}$ according to (2), and a possible extra contribution $\Lambda_{\mathrm{UV}}$ of size $\left|\Lambda_{\mathrm{UV}}\right| \sim$ $\left(E_{\mathrm{UV}}\right)^{4}$ from the unknown physics beyond the standard model. For definiteness, we also assume that $\epsilon_{\mathrm{var}}(q)$ contains only even powers of $q$ and recall that $q^{2}$ is defined by (1c) in terms of the three-form gauge field $A$ entering the field strength (1b). Allowing for a general even function $\epsilon(q)$ instead of the single Maxwell-type term $\frac{1}{2} q^{2}$ considered in the previous literature [6, 7, 8, 9, 10] will turn out to be an important ingredient for the cancellation of $\Lambda_{\text {bare }}$ values of arbitrary sign.

The generalized Maxwell and Einstein equations from action (1a) have been derived in Ref. 4]. The generalized Maxwell equation reads

$$
\nabla_{\alpha}\left(\sqrt{-\operatorname{det} g} \frac{F^{\alpha \beta \gamma \delta}}{q}\left(\frac{\partial \epsilon(q)}{\partial q}+R \frac{\partial K(q)}{\partial q}\right)\right)=0
$$

and reproduces the known equation [6, 7] for the special case $\epsilon(q)=\frac{1}{2} q^{2}$ and $\partial K / \partial q=0$. The first integral of (4) with integration constant $\mu$ and the final version of the generalized Einstein equation then give the following generic equations [4]:

$$
\begin{aligned}
\frac{\partial \epsilon(q)}{\partial q}+R \frac{\partial K(q)}{\partial q}= & \mu, \\
2 K\left(R^{\alpha \beta}-g^{\alpha \beta} R / 2\right)= & -2\left(\nabla^{\alpha} \nabla^{\beta}-g^{\alpha \beta} \square\right) K(q) \\
& +(\epsilon(q)-\mu q) g^{\alpha \beta}-T_{\mathrm{SM}}^{\alpha \beta},
\end{aligned}
$$

where $T_{\mathrm{SM}}^{\alpha \beta}$ is the energy-momentum tensor corresponding to the effective Lagrangian appearing in (1a) and (2). From general coordinate invariance, the energymomentum tensor is known to have a vanishing covariant divergence, $\nabla_{\alpha} T_{\mathrm{SM}}^{\alpha \beta}=0$.

For the special case $K(q)=K_{0}=$ const, (5b) reduces to the standard Einstein equation of general relativity. For the general case $d K / d q \neq 0$, the action (1a) and the resulting field equation (5b) correspond to those of Brans-Dicke theory [17, but without kinetic term for the scalar degree of freedom $\left(\omega_{\mathrm{BD}}=0\right)$. See also the related work on inflation theory [18, darkenergy models [19, 20, 21, 22, and the connection to $q$-theory [23, 24, 25].

The crucial difference between our theory and conventional $f(R)$ modified-gravity theories [18, 19, 20, 21, 22 lies in the appearance, for us, of the integration constant $\mu$ after integration over the three-form gauge field $A$, i.e., after solving the generalized Maxwell equation (4). As a result, the vacuum energy density entering the generalized Einstein equation (5b) is not the original vacuum energy density $\epsilon(q)$ from the action (1a) but the combination

$$
\rho_{V}(q) \equiv \epsilon(q)-\mu q .
$$

This gravitating vacuum energy density becomes a genuine cosmological constant $\bar{\Lambda} \equiv \Lambda(\bar{q})=\rho_{V}(\bar{q})$ for a spacetime-independent vacuum variable $\bar{q}$.

The field equations $(5 \mathrm{bb})$ can now be seen to have a Minkowski-type solution with spacetime-independent fields. For standard global spacetime coordinates, the fields of this constant solution are given by

$$
\begin{aligned}
g_{\alpha \beta}(x) & =\eta_{\alpha \beta}, \\
F_{\alpha \beta \gamma \delta}(x) & =q_{0} \epsilon_{\alpha \beta \gamma \delta}, \\
\psi(x) & =\psi_{0},
\end{aligned}
$$


with spacetime-independent parameters $\mu_{0}$ and $q_{0}$ determined by the following two conditions:

$$
\begin{gathered}
{\left[\frac{\mathrm{d} \epsilon(q)}{\mathrm{d} q}-\mu\right]_{\mu=\mu_{0}, q=q_{0}}=0,} \\
{[\epsilon(q)-\mu q]_{\mu=\mu_{0}, q=q_{0}}=0 .}
\end{gathered}
$$

Conditions (8a) and (8b) follow from (5a) and (5b), respectively, for $R=R^{\alpha \beta}=T_{\mathrm{SM}}^{\alpha \beta}=0$ and spacetimeindependent $q_{0}$.

The two conditions $8 \mathrm{ab}-8 \mathrm{~b}$ can be combined into a single equilibrium condition for $q_{0}$ :

$$
\Lambda_{0} \equiv\left[\epsilon(q)-q \frac{\mathrm{d} \epsilon(q)}{\mathrm{d} q}\right]_{q=q_{0}}=0
$$

with the derived quantity 26

$$
\mu_{0}=\left[\frac{\mathrm{d} \epsilon(q)}{\mathrm{d} q}\right]_{q=q_{0}} .
$$

The spacetime independence of $q_{0}$ implies that of $\mu_{0}$ in (10) and, with (5a), guarantees that the generalized Maxwell equation (4) is automatically solved by the Minkowski-type solution (77); see below for a general discussion of this important point. In order for the Minkowski vacuum to be stable, there is the further condition:

$$
\left(\chi_{0}\right)^{-1} \equiv\left[q^{2} \frac{\mathrm{d}^{2} \epsilon(q)}{\mathrm{d} q^{2}}\right]_{q=q_{0}}>0,
$$

where $\chi$ corresponds to the isothermal vacuum compressibility [3]. In the equilibrium vacuum relevant to our Universe, the gravitational constant $K\left(q_{0}\right)$ of the action (1a) can be identified with $K_{0} \equiv 1 /\left(16 \pi G_{N}\right)$ in terms of Newton's constant $G_{N}$.

Equation (9) corresponds to the first of the two constant-field equilibrium conditions given by Weinberg [1] as Eqs. (6.2) and (6.3): $\partial \mathcal{L} / \partial g_{\alpha \beta}=0$ and $\partial \mathcal{L} / \partial \phi=0$, having restricted the discussion here to the case of a single fundamental scalar field $\phi$. These two conditions turn out to be inconsistent, unless the potential term in $\mathcal{L}(\phi)$ is fine-tuned [1. See also Sec. 2 of Ref. 2 for further discussion on the impossibility of finding a natural Minkowski-type solution from the adjustment of a fundamental scalar field.

The crucial difference between a fundamental scalar field $\phi$ and our vacuum variable $q$ (a non-fundamental scalar field) is that the equilibrium condition for $q$ is relaxed: we find, instead of the condition $\partial \mathcal{L} / \partial q=0$,
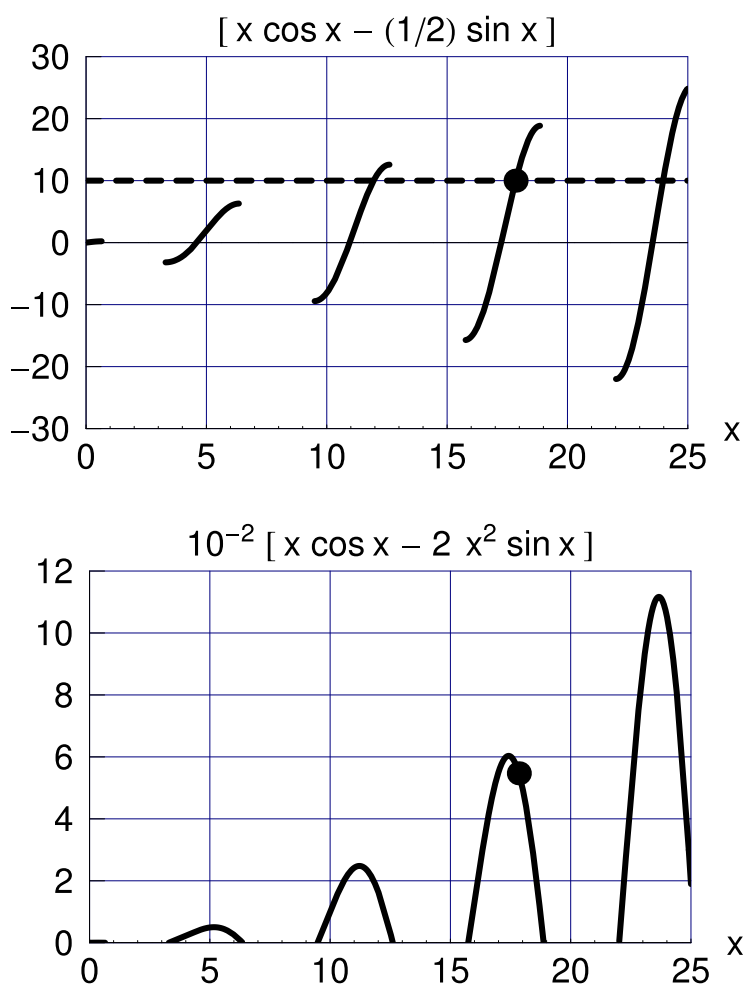

Fig. 1 Minkowski equilibrium vacua for a particular choice of the vacuum energy density function as given by (12). The curves of the top panel show the lefthand side of (13a) for those values of $x \equiv \widehat{q}^{2}$ that obey the stability condition (13b). The curves of the bottom panel show the corresponding positive segments of the inverse of the dimensionless vacuum compressibility $\widehat{\chi}$ defined by the left-hand side of (13b), the general dimensional quantity being defined by (11). Minkowskitype vacua (7) are obtained at the intersection points of the curves of the top panel with a horizontal line at the value $\lambda \equiv \Lambda_{\text {bare }} /\left(E_{\mathrm{UV}}\right)^{4}$ [for example, the dashed line at $\lambda=10$ gives the value $\left(\widehat{q}_{0}\right)^{2} \approx 17.8453$ corresponding to the heavy dot in the top panel]. Each such vacuum is characterized, in part, by the corresponding value of the inverse vacuum compressibility from the bottom panel [for example, $1 / \widehat{\chi}_{0} \approx 546.974$ shown by the heavy dot for the case chosen in the top panel]. Minkowski vacua with positive compressibility are stable and become attractors in a dynamical context (cf. Sec. 3 and Fig. 2).

the conditions $\nabla_{\alpha}(\partial \mathcal{L} / \partial q)=0$, which allow for having $\partial \mathcal{L} / \partial q=\mu$ with an arbitrary constant $\mu$. As a result, the equilibrium conditions for $g_{\alpha \beta}$ and $q$ can be consistent without fine-tuning. The approach based on such a $q$-variable bypasses the apparent no-go theorem (as foretold by Ftn. 8 of Ref. [1]) and formally solves the cosmological constant problem (as formulated in Sec. 2 of Ref [2]): the original action is not fine-tuned and 
need not vanish at the stationary point, but there still exists a Minkowski-type solution of the field equations. This realization that the $q$-field allows for a relaxation of the equilibrium condition is the first of the two most important new results of the present Letter.

The Minkowski-type solution of theory (1) is given by the fields (7) with a constant $q_{0}$ parameter that solves (9) and satisfies (11). At this moment, it may be instructive to work out a concrete example. A particular choice for the vacuum energy density function (3) is given by:

$$
\epsilon(q)=\Lambda_{\text {bare }}+(1 / 2)\left(E_{\mathrm{UV}}\right)^{4} \sin \left[q^{2} /\left(E_{\mathrm{UV}}\right)^{4}\right],
$$

which contains higher-order terms in addition to the standard quadratic term $\frac{1}{2} q^{2}$. Needless to say, many other functions $\epsilon(q)$ can be chosen, the only requirement being that the equilibrium and stability conditions can be satisfied [3]. With (12), the expressions for the equilibrium condition (9) and the stability condition (11) become

$$
\begin{array}{r}
\widehat{q}^{2} \cos \left(\widehat{q}^{2}\right)-(1 / 2) \sin \left(\widehat{q}^{2}\right)=\lambda, \\
\widehat{\chi}^{-1} \equiv \widehat{q}^{2} \cos \left(\widehat{q}^{2}\right)-2 \widehat{q}^{4} \sin \left(\widehat{q}^{2}\right)>0
\end{array}
$$

where $E_{\mathrm{UV}}$ has been used to define dimensionless quantities $\widehat{q} \equiv q /\left(E_{\mathrm{UV}}\right)^{2}$ and $\lambda \equiv \Lambda_{\text {bare }} /\left(E_{\mathrm{UV}}\right)^{4}$. A straightforward graphical analysis (Fig. 1) shows that, for any $\lambda \in \mathbb{R}$, there are infinitely many values $\widehat{q}_{0} \in \mathbb{R}$ which obey both (13a) and (13b). The top panel of Fig. 1 also shows that the $\widehat{q}$ values on the one segment singledout by the heavy dot already allow for a complete cancellation of any $\Lambda_{\text {bare }}$ value between $-15\left(E_{\mathrm{UV}}\right)^{4}$ and $+18\left(E_{\mathrm{UV}}\right)^{4}$.

\section{MINKOWSKI ATTRACTOR}

The cancellation mechanism discussed in the previous section provides the following general lesson. The Minkowski-type solution (7) appears without finetuning of the parameters of the action, precisely because the vacuum is characterized by a constant derivative of the vacuum field rather than by a constant value of the vacuum field itself. As a result, the parameter $\mu_{0}$ emerges in (8a) as an integration constant, i.e., as a parameter of the solution rather than a parameter of the Lagrangian. The idea that the constant derivative of a field may be important for the cosmological constant problem has been suggested earlier by Dolgov [27, 28] and Polyakov [29, 30, where the latter explored the analogy with the Larkin-Pikin effect 31] in solid-state physics.
However, instead of the fine-tuning problem of the cosmological constant, we now have the fine-tuning problem of the integration constant, namely, the chemical potential $\mu=\mu_{0}$ that fixes the value $q=q_{0}$ of the Minkowski equilibrium vacuum (or vice versa, $q_{0}$ fixing $\left.\mu_{0}\right)$. Any other choice of the integration constant $\left(\mu \neq \mu_{0}\right)$ leads to a de-Sitter-type solution [4. Still, it is possible to show that these de-Sitter-type solutions become dynamically unstable in a generalization of $q-$ theory and that the Minkowski equilibrium vacuum becomes an attractor.

For that purpose, we start from the realization of $q-$ theory in terms of a vector field $A_{\beta}(x)$ as discussed by Dolgov [28] or, equivalently, in terms of an aether-type velocity field $u_{\beta}(x)$ as discussed by Jacobson 32 . The constant vacuum field $q$ then appears [3] as the derivative of a vector field in the specific solution $u_{\beta}=\bar{u}_{\beta}$ corresponding to the equilibrium vacuum, $q g_{\alpha \beta} \equiv \nabla_{\alpha} \bar{u}_{\beta} \equiv$ $\bar{u}_{\alpha \beta}$. In this realization, the effective chemical potential $\mu \equiv d \epsilon(q) / d q$ plays a role only for the equilibrium states (i.e., for their thermodynamical properties), but $\mu$ does not appear as an integration constant for the dynamics. Hence, the fine-tuning problem of the integration constant is overcome, simply because there is no integration constant.

The instability of the de-Sitter solution has been demonstrated by Dolgov 28] for the simplest quadratic choices of the Lagrange density of $u_{\beta}(x)$ and for an energy scale $E_{\mathrm{UV}}=E_{\text {Planck }}$ entering $\Lambda_{\text {bare }}$ of (3) . (At this moment, we do not consider the possibility of having a variable gravitational coupling parameter, so that we set $K\left[u_{\alpha \beta}\right]=K_{0}=$ const.) For a spatially flat RobertsonWalker metric with cosmic time $t$ and scale factor $a(t)$, the initial de-Sitter-type universe evolves towards Minkowski spacetime by the following $t \rightarrow \infty$ asymptotic solution for the aether-type field $u_{\beta}=\left(u_{0}, u_{b}\right)$ and the Hubble parameter $H \equiv[d a / d t] / a$ :

$$
u_{0}(t) \rightarrow q_{0} t, \quad u_{b}(t)=0, \quad H(t) \rightarrow 1 / t,
$$

where $u_{0}(t)$ increases linearly with $t$ for constant $q_{0}$ (the norm of the vector field $u_{\beta}$ is taken 3 to be unconstrained, different from Ref. 32]). Figure 2 shows explicitly the attractor behavior, with the numerical value of $q_{0}$ in (14) appearing dynamically.

The following three remarks may help to better understand the role of (14). First, observe that, for finite values of $t$, the aether-type field approaching (14) does not correspond to the $q$-theory Ansatz, $u_{\alpha \beta} \equiv \nabla_{\alpha} u_{\beta} \neq$ $q g_{\alpha \beta}$ for $t<\infty$. Second, the fact that $H(t)$ in (14) and Figure 2 drops to zero means that the RobertsonWalker metric approaches the one of Minkowski space- 
time, whereas, for a positive asymptotic value of $H(t)$, the metric would have approached the one of de-Sitter spacetime. Third, the simple model with solution (14) does not appear to give a realistic description of the present Universe [33] and requires an appropriate modification (possibly implementing chameleon-type effects 34]), but, for the present discussion, the simple model suffices.

It is straightforward to show that the asymptotic solution (14) also holds for the generalized Lagrangian with a generic function $\epsilon\left(u_{\alpha \beta}\right)$ replacing the quadratic term, as discussed in Sec. V D of Ref. 3. At large cosmic times $t$, the curvature terms decay as $|R| \sim H^{2} \sim$ $1 / t^{2}$ and the Einstein equations lead to the nullification of the energy-momentum tensor of the $u_{\beta}$ field: $T_{\alpha \beta}[u]=0$. Since the exact expressions on the righthand-sides of (14) with $d u_{0} / d t=H u_{0}$ satisfy the $q-$ theory Ansatz $u_{\alpha \beta}=q g_{\alpha \beta}$, the energy-momentum tensor is completely expressed by the single constant $q$ : $T_{\alpha \beta}(q)=[\epsilon(q)-q d \epsilon(q) / d q] g_{\alpha \beta}$. As a result, the equation $T_{\alpha \beta}(q)=0$ leads to the equilibrium condition (9) for the Minkowski vacuum and to the equilibrium value $q=q_{0}$ in (14). This demonstrates that the compensation of a large initial vacuum energy density can occur dynamically and that Minkowski spacetime can emerge spontaneously, without setting a chemical potential. In other words, an "existence proof" has been given for the conjecture that the appropriate Minkowski value $q_{0}$ can result from an attractor-type solution of the field equations. The only condition for the Minkowski vacuum to be an attractor is a positive vacuum compressibility (11). This existence proof is the second of the two most important new results of the present Letter.

In the previous discussion, we illustrated the compensation of the "bare" vacuum energy density by use of the simplest realizations of the constant vacuum field $q$, where $q$ follows from derivatives of either the fundamental gauge field $A_{\beta \gamma \delta}(x)$ or the fundamental vector field $u_{\beta}(x)$. The constant vacuum field $q$ from the fourform field strength tensor (1b) has been discussed earlier in, e.g., Refs. 9, 10]. But these references consider a quadratic function $\epsilon(F)$, which can only compensate a $\Lambda_{\text {bare }}$ value of a particular sign. Our approach is generic and does not depend on the particular realization of the "quinta essentia" - the field $q$ which describes the deep (ultraviolet) quantum vacuum [35]. The only requirement for $q$ is that it must be a Lorentz-invariant conserved (i.e., spacetime-independent) quantity in flat Minkowski spacetime. In addition, an almost arbitrary function $\epsilon(q)$ allows for the cancellation of $\Lambda_{\text {bare }}$ values
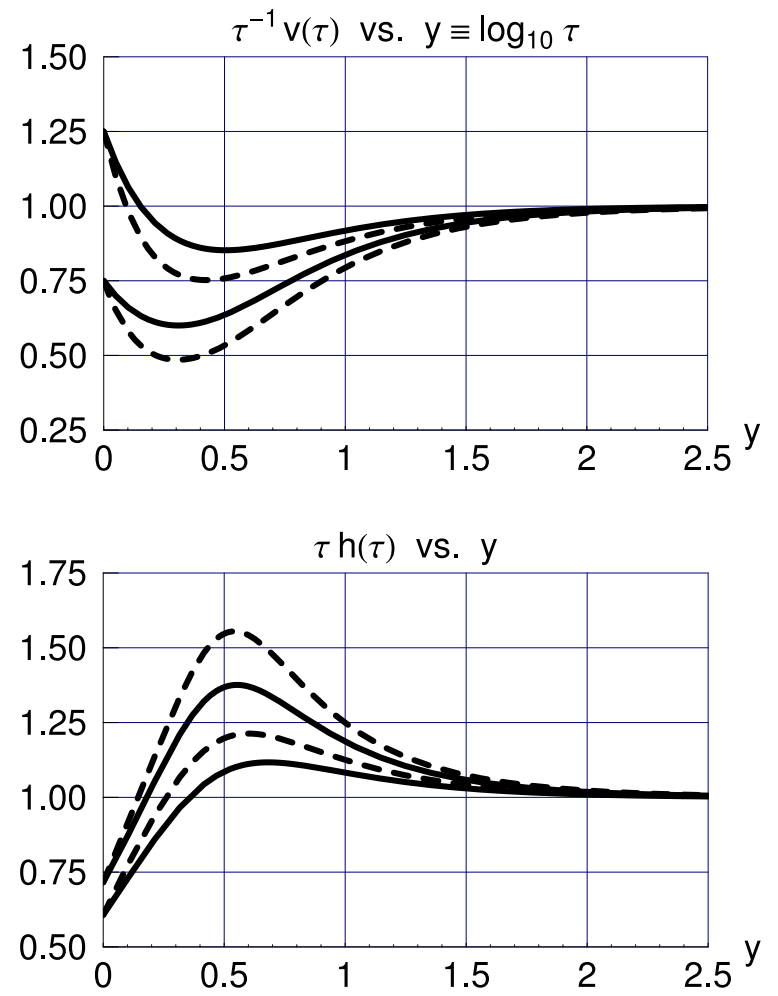

Fig. 2 Aether-field evolution and Minkowski attractor in a spatially flat Friedmann-Robertson-Walker universe with $\lambda \equiv \Lambda_{\text {bare }} /\left(E_{\text {Planck }}\right)^{4}=2$. Top panel: dimensionless aether-type field component $v \equiv$ $u_{0} / E_{\text {Planck }}$ [multiplied by a factor $\tau^{-1}$ ] plotted against the logarithm of the dimensionless cosmic time $y \equiv$ $\log _{10} \tau \equiv \log _{10}\left(t E_{\text {Planck }}\right)$. Bottom panel: dimensionless Hubble parameter $h \equiv H / E_{\text {Planck }}$ [multiplied by a factor $\tau$ ] plotted against $y$. The field equations are given by Eqs. (5) and (8) in Ref. 28] for $\rho_{\mathrm{vac}}=\Lambda_{\text {bare }}$ and $\eta_{0}=$ $-1: \ddot{v}+3 h \dot{v}-3 h^{2} v=0$ and $6 h^{2}=2 \lambda-(\dot{v})^{2}-3(h v)^{2}$, with the overdot standing for differentiation with respect to $\tau$. The four numerical solutions shown have boundary conditions $v(1)=1 \pm 0.25$ and $\dot{v}(1)= \pm 0.25$, with the dashed curves referring to negative $\dot{v}(1)$ [note that the top panel plots $v(\tau) / \tau$, not $v(\tau)$ ]. All four numerical solutions approach the Minkowski-spacetime solution (14) with, for the chosen model parameter $\Lambda_{\text {bare }} /\left(E_{\text {Planck }}\right)^{4}=2$, a value $q_{0} /\left(E_{\text {Planck }}\right)^{2}=1$ appearing dynamically [see top panel]. In fact, the same $t \rightarrow \infty$ asymptote is found for all boundary conditions $|v(1)-1| \leq 0.25$ and $|\dot{v}(1)| \leq 0.25$, which shows that (14) is a positive attractor. For the theory considered [defined by Eq. (2) of Ref. 28] for $\eta_{0}=-1$ ], the Minkowski vacuum is an attractor because the vacuum compressibility (11) is positive, $\chi\left(q_{0}\right)>0$.

of both signs; see, in particular, the example (12) discussed above. 
Finally, it may be of interest to compare our possible solution of the cosmological constant problem with that of the unimodular theory of gravity (see, e.g., Refs. 1, 37, 38, and further references therein). From the unimodular theory of gravity, the cosmological constant of standard general relativity is obtained as an integration constant and the Minkowski solution also follows without fine-tuning of the parameters of the action. As a purely classical theory, unimodular gravity is equivalent to general relativity, but its extension to the quantum world can be expected to be different from that of general relativity, which is at the core of our approach [the $q$ dependence of the gravitational coupling $K$ in action (1a) is not essential to obtain (9) and the particular aether-type solution discussed in the present section already has constant $K]$. Furthermore, the unimodular gravity theory would not allow for a spacetimedependent "cosmological constant" and, a forteriori, would not give an attractor-type solution approaching Minkowski spacetime.

\section{SUMMARY AND OUTLOOK}

In this Letter, we have shown that it is possible to find an extension of the current theory of elementary particle physics (the standard model), which allows for a Minkowski-spacetime solution with constant fields, without fine-tuning the extended theory in any way or shape. For this suggested solution, the cosmological constant $\Lambda_{\text {bare }}$ from (3), which includes the zeropoint energy $\Lambda_{\mathrm{SM}} \sim \pm\left(E_{\text {ew }}\right)^{4}$ of the standard model fields, is completely compensated by the $q$-field that describes the degrees of freedom of the deep quantum vacuum with energy scale $E_{\mathrm{UV}} \gg E_{\text {ew }}$.

This solves the main cosmological constant problem [39] and even addresses the next question (also raised in Ref. [1]): why is our present Universe close to the Minkowski vacuum or, in other words, why does Nature prefer flat spacetime? The answer to this question appears to be: because the Minkowski equilibrium state is an attractor and the Universe is moving towards it. We are close to this attractor, simply because our Universe is old.

There remain, however, other problems. Observational cosmology (see, e.g., Refs. [40, 41, 42] and further references therein) suggests a tiny remnant vacuum energy density $\rho_{V}$ of the order of $10^{-11} \mathrm{eV}^{4}$. This, then, leads to the additional cosmic coincidence problem: why is the nonzero vacuum energy density of the same order as the present matter energy density? One possible solution [5] of the cosmic coincidence problem may be related to quantum-dissipative effects during the cosmological evolution of the microscopic field $q(x)$. In any case, $q$-theory transforms the standard cosmological constant problem into the search for the proper decay mechanism of the vacuum energy density.

\section{ACKNOWLEDGMENTS}

It is a pleasure to thank A. Dolgov, L. Smolin, and M. Veltman for helpful comments on an earlier version of this Letter. GEV is supported in part by the Academy of Finland, Centers of Excellence Program 2006-2011 and the Khalatnikov-Starobinsky leading scientific school (Grant No. 4899.2008.2).

1. S. Weinberg, "The cosmological constant problem," Rev. Mod. Phys. 61, 1 (1989).

2. S. Weinberg, "Theories of the cosmological constant," in: N. Turok, Critical Dialogues in Cosmology (World Scientific, Singapore, 1997), p. 195, arXiv:astro-ph/9610044.

3. F.R. Klinkhamer and G.E. Volovik, "Self-tuning vacuum variable and cosmological constant," Phys. Rev. D 77, 085015 (2008), arXiv:0711.3170

4. F.R. Klinkhamer and G.E. Volovik, "Dynamic vacuum variable and equilibrium approach in cosmology," Phys. Rev. D 78, 063528 (2008), arXiv:0806.2805.

5. F.R. Klinkhamer and G.E. Volovik, "Vacuum energy density kicked by the electroweak crossover," Phys. Rev. D 80, 083001 (2009), arXiv:0905.1919.

6. M.J. Duff and P. van Nieuwenhuizen, "Quantum inequivalence of different field representations," Phys. Lett. B 94, 179 (1980).

7. A. Aurilia, H. Nicolai, and P.K. Townsend, "Hidden constants: The theta parameter of QCD and the cosmological constant of $N=8$ supergravity," Nucl. Phys. B 176, 509 (1980).

8. S.W. Hawking, "The cosmological constant is probably zero," Phys. Lett. B 134, 403 (1984).

9. M. Henneaux and C. Teitelboim, "The cosmological constant as a canonical variable," Phys. Lett. B 143, 415 (1984).

10. M.J. Duff, "The cosmological constant is possibly zero, but the proof is probably wrong," Phys. Lett. B 226, 36 (1989).

11. M.J. Duncan and L.G. Jensen, "Four-forms and the vanishing of the cosmological constant," Nucl. Phys. B 336, 100 (1990).

12. R. Bousso and J. Polchinski, "Quantization of fourform fluxes and dynamical neutralization of the cosmological constant," JHEP 0006, 006 (2000), arXiv:hep-th/0004134. 
13. A. Aurilia and E. Spallucci, "Quantum fluctuations of a 'constant' gauge field," Phys. Rev. D 69, 105004 (2004), arXiv:hep-th/0402096.

14. Z.C. Wu, "The cosmological constant is probably zero, and a proof is possibly right," Phys. Lett. B 659, 891 (2008), arXiv:0709.3314

15. M. Veltman, Diagrammatica: The Path to Feynman rules (Cambridge University Press, Cambridge, England, 1994), App. E.

16. N.D. Birrell and P.C.W. Davies, Quantum Fields in Curved Space (Cambridge University Press, Cambridge, England, 1982).

17. C. Brans and R.H. Dicke, "Mach's principle and a relativistic theory of gravitation," Phys. Rev. 124, 925 (1961).

18. A.A. Starobinsky, "A new type of isotropic cosmological models without singularity," Phys. Lett. B 91, 99 (1980).

19. W. Hu and I. Sawicki, "Models of $f(R)$ cosmic acceleration that evade solar-system tests," Phys. Rev. D 76, 064004 (2007), arXiv:0705.1158.

20. S.A. Appleby and R.A. Battye, "Do consistent $f(R)$ models mimic general relativity plus $\Lambda$ ?," Phys. Lett. B 654, 7 (2007), arXiv:0705.3199.

21. A.A. Starobinsky, "Disappearing cosmological constant in $f(R)$ gravity," JETP Lett. 86, 157 (2007), arXiv:0706.2041

22. P. Brax, C. van de Bruck, A.C. Davis, and D.J. Shaw, " $f(R)$ gravity and chameleon theories," Phys. Rev. D 78, 104021 (2008), arXiv:0806.3415

23. F.R. Klinkhamer and G.E. Volovik, " $f(R)$ cosmology from q-theory," JETP Lett. 88, 289 (2008), arXiv:0807.3896

24. F.R. Klinkhamer and G.E. Volovik, "Gluonic vacuum, $q$-theory, and the cosmological constant," Phys. Rev. D 79, 063527 (2009), arXiv:0811.4347

25. F.R. Klinkhamer, "Gluon condensate, modified gravity, and the accelerating Universe," Phys. Rev. D 81, 043006 (2010), arXiv:0904.3276

26. There is no need, here, to dwell on the interpretation of $\mu_{0}$ as a chemical potential 3, 4. Still, it may be relevant for the future development of the theory that the obtained vacuum can be viewed as a self-sustained system existing at zero external pressure, $P_{\text {ext }}=0$, and that $8 \mathrm{~b}$ can be read as the integrated form of the thermodynamic Gibbs-Duhem equation, $-P=\epsilon-\mu q$, provided the identification $\mu=\mathrm{d} \epsilon / \mathrm{d} q$ and the pressureequilibrium condition $P=P_{\text {ext }}=0$ hold.

27. A.D. Dolgov, "Field model with a dynamic cancellation of the cosmological constant," JETP Lett. 41, 345 (1985),

28. A.D. Dolgov, "Higher spin fields and the problem of cosmological constant," Phys. Rev. D 55, 5881 (1997), arXiv:astro-ph/9608175.
29. (a) A.M. Polyakov, "Selftuning fields and resonant correlations in 2-D gravity," Mod. Phys. Lett. A 6, 635 (1991); (b) I. Klebanov and A.M. Polyakov, "Interaction of discrete states in two-dimensional string theory," Mod. Phys. Lett. A 6, 3273 (1991), arXiv:hep-th/9109032

30. A.M. Polyakov, private communication.

31. (a) A.I. Larkin and S.A. Pikin, "Phase transitions of first order close to the second order," Sov. Phys. JETP 29, 891 (1969); (b) J. Sak, "Critical behavior and compressible magnets," Phys. Rev. B 10, 3957 (1974).

32. T. Jacobson, "Einstein-aether gravity: A status report," in proceedings of: From Quantum to Emergent Gravity: Theory and Phenomenology, PoS QG-PH, 020 (2007), arXiv:0801.1547.

33. V.A. Rubakov and P.G. Tinyakov, "Ruling out a higher spin field solution to the cosmological constant problem," Phys. Rev. D 61, 087503 (2000), arXiv:hep-ph/9906239

34. J. Khoury and A. Weltman, "Chameleon cosmology," Phys. Rev. D 69, 044026 (2004), arXiv:astro-ph/0309411.

35. The general term "quintessence" from Ref. 36] has, over the years, become associated with a fundamental scalar field and the use of the slightly different term "quinta essentia" for the vacuum field $q$ is to avoid any possible misunderstanding.

36. R.R. Caldwell, R. Dave, and P.J. Steinhardt, "Cosmological imprint of an energy component with general equation-of-state," Phys. Rev. Lett. 80, 1582 (1998), arXiv:astro-ph/9708069.

37. J.J. van der Bij, H. van Dam, and Y.J. Ng, "The exchange of massless spin two particles," Physica 116A, 307 (1982).

38. L. Smolin, "The quantization of unimodular gravity and the cosmological constant problem," Phys. Rev. D 80, 084003 (2009), arXiv:0904.4841

39. As to the terminology of "main" cosmological constant problem, we refer to the required cancellation of a huge initial contribution to the gravitating vacuum energy density which is of order $\left(E_{\text {ew }}\right)^{4}$ or more; see also the discussion under Eq. (3).

40. A.G. Riess et al. [Supernova Search Team Collaboration], "Observational evidence from supernovae for an accelerating universe and a cosmological constant," Astron. J. 116, 1009 (1998), arXiv:astro-ph/9805201

41. S. Perlmutter et al. [Supernova Cosmology Project Collaboration], "Measurements of $\Omega$ and $\Lambda$ from 42 highredshift supernovae," Astrophys. J. 517, 565 (1999), arXiv:astro-ph/9812133.

42. E. Komatsu et al., "Five-year Wilkinson Microwave Anisotropy Probe (WMAP) observations: Cosmological interpretation," Astrophys. J. Suppl. 180, 330 (2009), arXiv:0803.0547 